\title{
MENTI MIGRANTI, MENTI ADOLESCENTI. AVANZARE SUL MARGINE: DAL TRAUMA IMPENSABILE ALLE PAROLE DEL DOLORE
}

\author{
Migrant minds, adolescent minds. Advancing on the margin: \\ from the unthinkable trauma to the words of pain
}

Virginia De Micco*

\begin{abstract}
Riassunto. Vengono esaminati i nodi traumatici connessi alla migrazione e allo sradicamento soprattutto nelle situazioni adolescenziali. In particolare vengono analizzate le memorie traumatiche e le trasformazioni dei vissuti corporei nei transiti migratori. Infine una particolare attenzione è dedicata alle complesse dinamiche relazionali che si stabiliscono nei centri di accoglienza dove spesso gli operatori si ritrovano a 'raccogliere' nelle loro menti gli elementi traumatici insostenibili che i migranti portano con sé.
\end{abstract}

Parole Chiave: migrazioni; adolescenza; memorie traumatiche; testimonianza.

\begin{abstract}
The article examines traumatic nodes related to migration and rootlessness, especially in adolescent situations. In particular the traumatic memories and the transformations of body experiences in the migratory transits are analyzed. Finally, particular attention is given to the complex relational dynamics that are established in accommodation centers where operators are often "collecting" in their minds unsustainable traumatic elements that immigrants bring with them.
\end{abstract}

Keywords: migrations; adolescence; traumatic memories; testimony.

\section{Crescere senza frontiera}

II materiale trattato nel presente articolo proviene da interventi di supervisione gruppale e di sostegno individuale svolti presso un centro di accoglienza per minori non accompagnate rivolti sia all'équipe di operatori che alle giovani ospiti del centro.

Società Psicoanalitica Italiana - IPA. Caserta, Italia. E-mail: vdm6396@virgilio.it. Orcid: 00000003-1376-9049. 
Vomitata dalla terra: così mi sentivo, racconta Okoko, ma raccontare è troppo, forse neanche dire rende l'idea, è una specie di singulto o forse addirittura un 'conato', uno sforzo di mettere fuori qualcosa, misto singolare di rigetto e però anche di espressione (ex-premere, mettere fuori). E' arrivata in un paesino italiano attraverso la rotta libico-centrafricana, proveniente dalla Nigeria, ha un'età in un certo senso indefinibile: è una ragazzina, 16 anni dichiarati, se la guardi profondamente negli occhi sembra ancora a tutti gli effetti una bambina, una bambina sperduta, se la vedi muoversi tra le stanze del centro di accoglienza sembra una donna fatta, esperta, a tratti decisamente prepotente che sa come farsi rispettare, se provi a parlarle spesso ti ride in faccia, misto di sfida, di angosciosa impossibilità di raggiungerla, di terribile sfiducia, poi all'improvviso quando meno te l'aspetti fa irruzione qualcosa, una frase, un gesto, un'immagine il cui statuto psichico è tutto da decifrare, letteralmente da ri-collocare in una mente, in un apparato psichico, elementare e fondamentale apparato simbolizzante.

E ancora, subito dopo averla detta, quella 'cosa' resta là, in un certo senso inappropriabile proprio per chi I'ha pronunciata, 'gettata' forse perché un altro apparato psichico la raccolga e possa custodirla innanzi tutto e poi, dopo averla 'sofferta', metabolizzarla, farne senso. Tutto ciò costa un'enorme fatica, un lavoro che attraversa la carne e la mente, sostanza sensibile e pensante, soprattutto di chi ospita, forse transitoriamente ma necessariamente, pezzi sradicati e sradicanti. "Chi è sradicato sradica", scriveva Simone Weil (1990, p. 53), il contatto con chi porta su di sé i segni dello sradicamento traumatico non lascia indenni, continua incessantemente a 'sradicare' ciò che gli si accosta. La percezione diffusa e immediata che i 'migranti' (e dopo torneremo sull'ambiguità semantica di questo termine) portino 'disordine e confusione' traduce il contatto, altrettanto traumatico, con un elemento ignoto innanzi tutto perché ha smarrito la sua forma, la sua immagine e la sua stessa griglia di pensabilità, ignoto dunque innanzi tutto a sé stesso perché ha smarrito gli strumenti per presentarsi in quanto 'umano'.

Se la dimensione di una mente adolescente appare ormai sufficientemente acquisita in ambito psicoanalitico fino a diventare cifra interpretativa non solo di una particolare età della vita ma più in generale di una modalità di funzionamento psichico, cosa comporterà il tentativo di delineare la dimensione di una mente migrante? $\mathrm{E}$ in cosa le due dimensioni mostrano una omologia di funzionamento, fino a poter essere individuate come manifestazioni elettive di quei soggetti dislocati che la contemporaneità ci restituisce? Adolescenza e migrazione non descrivono solo 'esperienze' psichiche specifiche ma, ancora di più, scenari e configurazioni psichici incentrati necessariamente sul cambiamento e sulle trasformazioni. 
Come si ricorderà León e Rebeca Grinberg (1990) ritenevano che la migrazione costituisse una vera e propria esperienza di rinascita in cui viene riattivata una condizione assimilabile a quella infantile: ci si ritrova in una condizione di bisogno in cui si fa di nuovo l'esperienza di una corporeità dominata da necessità impellenti, si prova di nuovo lo spaesamento rispetto ad una lingua 'straniera' e sconosciuta in cui si riattiva potentemente la sensibilità agli aspetti emotivi e prosodici piuttosto che agli aspetti semantici della lingua, si vive di nuovo la dipendenza da relazioni che forniscano una essenziale funzione un accoglimento psichico, di rispecchiamento e di riconoscimento.

\section{Menti migranti}

Esperienza di 'rinascita' dunque, la quale coinvolge le basi stesse di quell'intelaiatura culturale che regge lo psichico rendendo addirittura necessario rifare un processo di simbolizzazione delle esperienze primarie, con tutto il rischio che tale operazione di ri-simbolizzazione psichica nel nuovo contesto non riesca e che ne derivino gravi difficoltà psichiche.

Questa condizione ci svela, d'altro canto, un livello di strutturale precarietà di tutte le nostre acquisizioni psichiche che possono inopinatamente essere rimesse in discussione dalle fondamenta in tali situazioni di vero e proprio 'stravolgimento' identitario: ci troviamo confrontati con una perdita radicale di traiettorie identificative che coinvolge addirittura le basi stesse della sensorialità e dell'affettività, forme elementari su cui costruire il senso di sé.

L'apparato culturale dunque non rappresenta solo una sorta di 'rivestimento' di funzionamenti psichici sottostanti ma va a costituire una vera e propria 'pelle' psicoculturale. Pertanto se questa pellicola protettiva e costitutiva si lacera andrà ricostituita in modi nuovi e inediti, con tutto il rischio che restino delle ferite psichiche non rimarginabili.

Le migrazioni comportano sempre un costo psichico elevatissimo costituito da un incessante lavoro di lutto, da una continua necessità di elaborare angosce persecutorie e depressive e di decodificare costantemente situazioni 'enigmatiche' sul piano affettivo e simbolico, esperienza che si rinnova e si ripresenta 'quotidianamente' alla 'mente migrante'. Questo specifico aspetto di un traumatismo diffuso e pervasivo era già stato evidenziato da quel finissimo antesignano degli studi di psicopatologia dell'emigrazione e di etnopsichiatria che fu Michele Risso (1982), il quale descriveva il processo di assimilazione, come si preferiva indicarlo negli anni '70, o di 'integrazione', come è uso invalso oggi, come un autentico "microtraumatismo quotidiano" (Frigessi Castelnuovo, Risso, 1982). 
L'esperienza migratoria dunque è sempre stata segnata da una profonda traumaticità ${ }^{1}$ ma nella più stringente attualità, soprattutto nelle rotte che attraversano il Mediterraneo, è caratterizzata da una distruttiva violenza identitaria che ha effetti profondamente destrutturanti sulla psiche dal momento che ne vengono attaccate tutte le funzioni rappresentative e simbolizzanti, imponendo una massiccia mutilazione delle memorie collettive ed individuali ad esempio, è per questo che nella relazione coi migranti attualmente vengono particolarmente sollecitate le aree psichiche non rappresentate e non rappresentabili. II problema dunque non è più soltanto quello di decodificare e far dialogare differenze culturali quanto piuttosto di sporgersi costantemente su un 'inassimilabile', su un 'non rappresentabile', cercare dunque di avanzare sul margine di trasformazioni individuali e collettive dagli esiti in larga parte imprevedibili e impredicibili.

Le migrazioni nell'epoca della globalizzazione ci pongono davanti dunque a scenari in parte inediti rispetto alle consuete problematiche psichiche e relazionali connesse ai movimenti migratori, scenari che ci spingono a riflettere sulla configurazione di una vera e propria 'mente migrante', costretta costantemente a riformularsi sui margini piuttosto che ad abitare spazi.

\footnotetext{
1 La migrazione è sempre stata un "fenomeno sfuggente" con le parole di J-P Raison (1980), per la sua stessa natura fluida e mutevole bisogna prestare continua attenzione a non generalizzare visioni del fenomeno che dipendono in larga parte dal vertice scelto, dalla 'posizione' dell'osservatore, in molteplici sensi, osservatore che dunque non può vedere che uno spicchio di un fenomeno appunto così mutevole e complesso, da un lato, così strutturalmente 'sfuggente' dall'altro. Inoltre la migrazione è caratterizzata da un'estrema dinamicità e da accelerati mutamenti antropologici per cui ciò che vediamo e descriviamo in un dato momento può poi essere rapidamente modificato o addirittura sovvertito.

Non a caso si parla di 'flussi' migratori, evocando un fenomeno liquido e inarrestabile: appunto come un fluido che non ha forma propria, ma assume la forma che il recipiente che lo contiene contemporaneamente gli consente e gli impone. Dunque il 'territorio' di accoglienza (territorio storico e geografico naturalmente, territorio antropologico) decide in gran parte della 'forma' che esso può assumere: quello che un po' sbrigativamente chiamiamo 'fenomeno' migratorio è in realtà la risultante di un incontro tra diverse realtà antropologiche.

Insomma occorre conservare una continua attenzione sull'effetto ottico, per così dire, particolarmente forte in questo campo, in cui quando sembra di osservare qualcosa che appartiene all'oggetto, all'altro, soprattutto all'altro culturale, invece spesso stiamo osservando e parlando di qualcosa che appartiene al 'noi', ai nostri strumenti osservativi, ancora di più ai nostri stessi 'strumenti per pensare', ivi compresi i nostri strumenti istituzionali, quelli che ad es. siamo in grado di mettere in campo per affrontare le emergenze.

Proprio nell'immaginare che siano interamente pensati per l'altro mettono, invece, più immediatamente a nudo il nostro modo di 'costruircelo' l'altro, appunto, e non dobbiamo mai dimenticare che implicitamente e preliminarmente stiamo chiedendo/imponendo a questo altro di infilarsi dentro quella 'griglia' di pensiero e di percezione attraverso cui possiamo rappresentarcelo. Inutile dire che tale griglia è costituita non solo da elementi consci e manifesti ma ancor più da elementi inconsci e impliciti, ancor più costrittivi e addirittura 'prescrittivi', come possiamo ben intuire, ed è proprio su questi aspetti impliciti veicolati dai funzionamenti istituzionali che occorre, tra gli altri, interrogarsi.
} 
Condizione per certi versi assimilabile a quella della 'mente adolescente': non a caso due participi presenti caratterizzano queste formulazioni che cercano di tradurre delle particolari condizioni psichiche ed esistenziali in cui si è sospesi in una trasformazione forse senza approdo.

\section{Menti adolescenti}

La radicale ristrutturazione identitaria dell'adolescenza si innesta su quella vera e propria rivoluzione somatica che è connessa alla pubertà, ma forse non esiste un'altra età della vita più intensamente modellata dall'esperienza culturale quanto quella adolescenziale, anzi in un certo senso l'intera 'adolescenza' può essere ritenuta una vera e propria 'invenzione' culturale nel senso specifico che più si allarga il bagaglio culturale da apprendere tanto più si dilata il tempo dell'adolescenza².

Tutto il peso culturale nella 'vicenda adolescente' è ben evidenziato proprio dal fatto che l'adolescenza come 'tempo critico' costituisce una esperienza specifica del contesto culturale moderno occidentale, fino a diventare addirittura cifra specifica del postmoderno e delle sue forme psicoantropologiche 'liquide' (Bauman, 2002, 2006), mentre si tratta di un tempo sostanzialmente sconosciuto ad altri contesti culturali, soprattutto a quelli extraoccidentali, o di una esperienza alla quale da pochissimo questi stessi contesti si stanno affacciando, con ricadute estremamente complesse sia sul piano dei tragitti individuali che sul piano delle dinamiche collettive.

Infatti la soluzione 'classica' adottata nei contesti extraoccidentali per segnare il passaggio dall'età infantile a quella adulta era rappresentata da varie forme più o meno strettamente codificate di riti di passaggio, così come sono stati identificati e definiti da Van Gennep (1909)³.

La oggettiva 'violenza' delle pratiche iniziatiche che hanno lo scopo di modificare e stabilizzare definitivamente 'I'identità' degli individui andava in qualche modo a 'sostituire' il lento e faticoso lavoro di lutto richiesto dal transito adolescenziale, transito peraltro dall'esito incerto e difficilmente predicibile: le angosce individuali connesse alla metamorfosi adolescenziale ricevono così una sorta di risposta predeterminata attraverso una azione rituale in cui gruppo e individuo si rinforzano reciprocamente. Questa peculiare

\footnotetext{
2 Si tratta infatti di un tempo di 'crescita'. Adolesco: cresco/imparo; non a caso nell'etimo latino I' 'accrescimento' sul piano fisico si intreccia con la necessità di crescere soprattutto sul piano sociale, di fornire dunque tutte le prestazioni che si richiedono all'individuo adulto.

3 Si tratta di rituali di iniziazione che hanno lo scopo specifico di allontanare in particolare i giovani maschi dall'aggregazione alla cerchia materno/infantile per associarli definitivamente a quella dei maschi adulti. Agli iniziati verranno richieste e riconosciute tutte le prerogative sociali, pubbliche e private, che spettano alla condizione adulta: in questo senso tutti i travagli psichici e i compiti evolutivi connessi alla fase adolescenziale vengono per così dire 'condensati' e risolti attraverso le vicissitudini iniziatiche.
} 
modalità di 'risoluzione' del transito adolescenziale tendeva a cancellare il 'tempo storico' di tale riformulazione identitaria, integralmente riassorbito nel tempo della ripetizione ritualizzata.

Tutti questi apparati rituali si dimostrano però insufficienti nel rispondere in maniera efficace ai nuovi bisogni psichici legati ai rapidi cambiamenti socioculturali cui tali popolazioni sono andate incontro.

Basti pensare che le stesse derive estremistiche ed integralistiche che coinvolgono tanti giovani occidentali migranti di seconda o terza generazione possono essere lette proprio in relazione al fallimento di tali meccanismi collettivi. Giovani sostanzialmente sradicati che si ritrovano a dover affrontare le profonde modificazioni strutturali legate all'adolescenza senza avere a disposizione nessuno strumento culturale efficace ${ }^{4}$. Da un lato gli apparati culturali tradizionali sono stati svalorizzati nei transiti migratori, dall'altro i modelli culturali occidentali sono stati 'imitati' solo superficialmente, cosicché nella crisi adolescenziale risulta particolarmente difficile, se non impossibile, per questi adolescenti fare appello a figure genitoriali introiettate che possano costituire validi supporti identificativi $i^{5}$.

Le 'riuscite' integrazioni dei bambini migranti svelano spesso tutto il loro vuoto identificativo sotto l'impatto del destrutturante smarrimento dell'adolescenza. Tali integrazioni si rivelano così molto spesso frutto solo di attitudini mimetiche, di imitazioni sul piano psichico, piuttosto che di autentiche introiezioni; 'mimesi' tanto massicce - e dunque tali da sostenere I'illusione di 'perfette' e funzionanti 'integrazioni' - quanto in realtà labili e, per così dire, 'revocabili' subitaneamente e sostituibili con 'mimesi' altrettanto radicali ma magari di segno opposto sul piano ideologico. Ecco come si può drammaticamente passare da 'rapper' a fondamentalista islamico nel giro di pochi anni o addirittura mesi.

L'adolescenza del resto costituisce un tempo di soggettivazione delle assegnazioni simboliche, nelle quali bisogna riuscire a 'collocarsi' trovando il proprio posto nell'ordine genealogico, nell'ordine delle proprie appartenenze simboliche.

Questa dimensione verticale, storica, che fonda le proprie origini in una memoria e restituisce un solido senso di appartenenza, è spesso smarrita e infranta negli adolescenti migranti, i quali possono ritrovarsi paradossalmente a cercare di ricostituire una sorta di 'purezza' identitaria per tentare di occultare

\footnotetext{
${ }^{4}$ Ovverosia nessuno strumento capace di fornire loro una simbolizzazione efficace sul piano psichico, capace di indicare il loro posto nell'ordine dei generi e delle generazioni

5 Ciò riguarda in particolare la figura e la dimensione psichica 'paterna' in quanto rappresentante e garante di una 'valido' universo simbolico.
} 
il vero e proprio buco che si è prodotto nel tessuto originario, dunque nello stesso fondamento della percezione di sè.

\section{Ferite o buchi? L'impossibile verità}

Il trauma migratorio è sempre stato un trauma occultato: nella lunga storia della psicopatologia della migrazione, che possiamo far cominciare con le osservazioni di Achille Foville figlio intorno alla fine del '700 nella città portuale di Le Havre, tale elemento traumatico è stato a lungo non riconosciuto dal sapere psichiatrico ma, ancora di più, si è tramutato in un 'disagio nascosto' per coloro stessi che lo hanno 'portato' per così dire. Tale caratteristica del trauma migratorio aveva spinto già Gianfausto Rosoli (1988) negli anni '70 a sottolineare che un vero bilancio del costo psichico delle migrazioni potesse essere svolto solo nell'arco di due o tre generazioni, mentre Norman Sartorius, in un rapporto all'OMS, segnalava come tali costi riguardino non solo chi parte ma anche chi resta, chi accoglie e chi ritorna. Tutto l'arco dell'esperienza migratoria, nello spazio ma anche nel tempo, è attraversato da un 'traumatico' tanto pervasivo quanto non riconosciuto, attraversato quindi da un dolore 'non pensato', da una perdita non rappresentabile non solo per coloro che partono ma anche per coloro che accolgono.

Nelle più recenti ondate migratorie come abbiamo evidenziato il trauma assume da un lato aspetti così massicci e destrutturanti da sospingere verso l'esperienza del 'disumano', evocando modalità che richiamano quelle dei genocidi, quelle cioè che hanno comportato storicamente la perdita dello statuto 'umano' per interi gruppi di popolazioni, dall'altro si traduce in un traumatismo continuato e pervasivo legato all'impossibilità di reperire adeguati strumenti di simbolizzazione dell'esperienza psichica. Questa instabilità dei referenti metapsichici e metasociali, come li definisce René Kaes (2008, 2009), determina la costituzione di ambienti 'umani' costantemente esposti al 'traumatico' piuttosto che ambienti capaci di proteggere psichicamente, proprio perché si tratta di contesti costitutivamente labili sul piano delle capacità simbolizzanti. E' proprio l'impatto di traumi massivi su ambienti simbolici fragili quello che la mente migrante configura. Ecco perché il trauma migratorio contemporaneo si gioca specificamente sullo scenario dell' 'attualità' in senso psichico ed è su questi scenari che va indagato.

Potrei sintetizzare questa complessa situazione con questa formula "più si cerca di indagare le ferite migratorie e più ci si ritrova invece davanti ai buchi che ha lasciato", buchi di senso e lacune nella memoria come unica possibilità di sopravvivenza psichica.

Già Ferenczi (1932, p. 131ss) segnalava come spesso l'esito di un grave evento traumatico comporti un "accomodamento con lacune", riferendosi ad 
una sorta di cicatrice psichica, di mutilazione di intere aree psichiche non più recuperabili. Ma è soprattutto la riflessione di Green (1996) sul negativo che ci apre la possibilità di pensare ad una attiva e massiccia operazione psichica di disinvestimento delle tracce traumatiche che esita nella formazione di una 'lacuna'. Quest'ultima modalità è certamente la più insidiosa, quella che tende a 'cancellare' le tracce dell'evento rendendolo addirittura non-esistito.

Sembrerebbe di trovarsi di fronte ad un estrema forma di "trasformazione autoplastica", con le parole di Ferenczi, in cui il disinvestimento si estende fino agli elementi affettivo-percettivi, in cui si arriva cioè a non percepire più il dolore non perché ci si rifugia dietro ad una 'corazza' protettiva ma, al contrario, perché non si oppone più alcuna resistenza. Come scrive Ferenczi (1932) infatti "di fronte a una lama che penetra si sente meno dolore se non si fa resistenza".

Più si immagina di dover lavorare in una logica restitutiva e riparativa, di ricucitura di tessuti psichici, ovverosia di legami e di storie, e più invece bisogna trovare uno spazio per lasciare in giacenza traumi ancora impensabili, spazi che hanno bisogno di restare anonimi per così dire, nel senso che bisogna ignorare a chi appartengano con precisione, chi possa farsene carico, fino a quando non potranno essere di nuovo assunti in prima persona. Tali spazi hanno bisogno spesso di essere 'ospitati' nella mente di un altro.

La prima domanda che il paese di accoglienza, ma forse meglio sarebbe dire di approdo, fa al migrante è appunto di 'identificarsi', dire chi è e 'raccontare' la sua storia. Ma questa richiesta ha un effetto decisamente perturbante dal momento che si chiede a chi ha smarrito del tutto la propria percezione identitaria di dire chi è, di assumere su di sé il proprio nome, che implica appartenenze e memorie, quando invece c'è bisogno di sospenderle; si chiede di raccordare passato e presente quando invece c'è bisogno di 'opacizzare' la propria storia (Sayad, 2001): assumerla come propria, riconoscersi in essa, attraversare le ferite e i lutti che comporta e la dolorosissima frattura con l'origine che mette in gioco, comporterà un lento e doloroso lavoro psichico di ri-soggettivazione che potrà anche non avvenire mai. Ecco perché il migrante nel rispondere a questa domanda cercherà soprattutto di intuire quale risposta desidera l'interlocutore, cercherà di diventare ciò che immagina l'altro voglia da lui in un vertiginoso gioco di specchi e proiezioni incrociate.

Ecco perché cercare di stabilire la 'verità' di queste storie, se siano compatibili o meno con la possibilità di ottenere uno statuto protetto come rifugiato ad esempio, rappresenta un vero e proprio slalom tra una sorta di 'copioni' dell' orrore, improvvise irruzioni di frammenti devastanti di immagini ri-vissute piuttosto che raccontate, talvolta in terza persona, quasi una sorta di bricolage psichico tra spezzoni di eventi vissuti sulla propria pelle o visti su quella di altri. Intreccio inestricabile tra verità e fiction in un certo senso, che 
non rappresenta una falsità ma una verità finzionale, la quale peraltro può costituire l'unica verità a cui si può dare espressione, poiché con le parole di Kafka quello che possiamo ex-premere, mettere fuori, è ciò che non siamo, la menzogna.

Si tratta di quella sorta di menzogna vitale che è possibile organizzare in un racconto di sé sostenibile: risultato assolutamente paradossale in cui magari non si può accedere a ciò che si è vissuto in prima persona, e tantomeno esporlo pubblicamente al giudizio e alla valutazione, non si può soggettivare ciò che si è soggettivamente subito perché significherebbe entrare in contatto con affetti devastanti incompatibili col mantenimento dello statuto di soggetto. Si può invece cercare di costruire una storia 'laterale', per così dire, una storia che forse non è proprio la tua ma è quello che altrettanto facilmente ti sarebbe potuto accadere, quello che saresti potuto essere: parti dislocate di soggetti frammentati, per i quali come si può ben intuire è l'angoscia di integrazione la più insostenibile. Ancora una volta i buchi identitari vengono colmati da narrazioni di sé finzionali: laddove le ferite sono troppo dolorose, restano congelate o 'murate' nel corpo, come memorie indelebili da un lato ma inattingibili dall'altro, dislocate in un fuori psiche come unico mezzo per conservarle.

Le esperienze di terrore che restano inassimilabili per la psiche dovranno allora rimanere confinate e congelate in un fuori-psiche, spesso si 'imprimeranno' nella carne, lì resteranno, fino a quando non potranno essere di nuovo pensate, trasformate di nuovo in esperienze 'umane', cosa che potrà anche non avvenire mai.

Piuttosto che 'incriptate' come memorie segrete, secondo la definizione di Abraham e Torok (1993), tali elementi traumatici impensati sono spesso 'nascosti in piena vista' per così dire, affioranti alla superficie piuttosto che nascoste in profondità, spesso come dicevamo letteralmente incorporate, impresse/espresse nel corpo.

Ecco perché i migranti sembrano chiedere a chi intende 'accoglierli' di poter sostare in questo statuto sospeso della soggettività, tollerare statuti identitari multipli e revocabili in cui possano convivere per un tempo aree disomogenee di sè. In particolare soprattutto gli operatori dei centri di accoglienza devono poter rimettere in gioco lo statuto transizionale della loro soggettività per accogliere, letteralmente per 'tenere dentro', ciò che il contatto con le menti migranti suscita. Poter tenere dentro una accanto all'altra, ad esempio, l'immagine delle brutali violenze subite da molte donne migranti con la consapevolezza della loro partecipazione al mercato della prostituzione, tollerandone questo vero e proprio doppio statuto identitario per un certo 
tempo, fino a quando sarà possibile avviarne una dolorosa ricomposizione attraverso una vera e propria riedificazione soggettiva.

\section{Il doppio corpo del migrante}

Il luogo dove tutte le dinamiche che abbiamo descritto si coagulano e si scaricano al massimo grado è sicuramente il corpo del migrante. La centralità dell'esperienza corporea e dei suoi cambiamenti percettivi e rappresentativi è cruciale sia in adolescenza che nei transiti migratori, col loro sommarsi e reduplicarsi negli adolescenti migranti.

Come abbiamo già segnalato da un lato il migrante precipita nuovamente in una condizione di bisogno corporeo estremo (fame, freddo, privazioni) e di dipendenza, quasi rivivendo lo stato infantile, dall'altro fa l'esperienza del suo corpo in termini di corpo estraneo nel nuovo contesto, esteticamente differente, capace di suscitare rigetto e curiosità, attraverso lo sguardo dell'altro fa una nuova esperienza di sé.

Sarà necessario dunque ricostituire completamente un'immagine di sé nel nuovo contesto con effetti la cui velocità può risultare addirittura sconcertante, soprattutto nell'esperienza più recente dei centri di accoglienza. Ho affrontato in altri lavori cui rimando le complesse relazioni che si stabiliscono in questi microcosmi interetnici (De Micco, 2019). Qui mi interessa solo evidenziare come una delle manifestazioni delle menti migranti sia costituita da questi corpi in transizione. Tutto ciò si rende specialmente evidente negli adolescenti, e soprattutto nelle adolescenti, migranti, in particolare minori non accompagnate, i cui corpi sembrano già parlare attraverso un codice che come soggetti non decifrano.

Così è sconcertante vedere quanto rapidamente, a poche settimane dallo sbarco sulle coste italiane, si dotano di cellulare e cuffiette perennemente indossate quasi a costituire un guscio psichico e allestiscono un profilo facebook con foto rigorosamente in pose da rotocalco, caricatura involontaria e a tratti grottesca dell'immaginario occidentale, mimesi impressionante nella sua adesività mutilante che cancella le storie e le appartenenze, ma forse proprio per questo così intensamente cercata: proprio per diventare 'altro' da sé il più rapidamente possibile, per liberarsi di quello che si è stati. Quando si comincia a ritornare 'umani' è terribile ripensare all'inumano in cui eri precipitato: se hai dormito per terra, hai mangiato rifiuti, hai subito violenze senza quasi più sentirle, è quasi insopportabile tornare ad una quotidianità 'ordinata' e cominciare a vedere con occhi atterriti quello che eri diventato.

Così, tornando alla storia da cui eravamo partiti, Okoko, assieme alla sua compagna di stanza Sonia, fa disperare le operatrici del centro di accoglienza: 
non sappiamo proprio come prenderle, sono pigre e non hanno voglia di fare nulla, dicono che sono sempre stanche, la mattina dormono fino a tardi e non vogliono andare al corso di italiano, ma poi ci andiamo caute perché sappiamo quello che hanno subito e quello che le aspetta appena usciranno di qui, quando compiono 18 anni anche a 18 anni e un giorno devono andare fuori di qui e da maggiorenni è ancora più difficile trattenerle, poi appena fuori ci sono le maman o i 'fidanzati' che le stanno già aspettando, queste ragazzine soprattutto le nigeriane le fanno venire tutte qui per prostituirsi, magari non glielo dicono chiaramente quando partono ma spesso le famiglie lo sanno e loro sanno che lo devono fare per le famiglie, quando le madri telefonano è terribile... all'inizio ci sembrava una cosa bellissima che potessero sentirsi a questa distanza dopo tutto quello che avevano passato... ma poi dopo le vedevamo molto turbate, poi abbiamo capito... perché le ricordano quello che devono fare.

Finalmente le due ragazze accettano di partecipare ad una manifestazione enogastronomica interetnica cucinando dei piatti tipici: si vestono con minigonna e tacchi alti, le operatrici pur notando l'inadeguatezza dell'abbigliamento non dicono nulla - misto di aggressività e di impotenza - ma quando arrivano sul posto Okoko sembra guardarsi per la prima volta con gli occhi degli altri, il disagio che le impongono quei tacchi alti che evidentemente aveva immaginato l'avrebbero fatta sentire 'all'altezza', diventa talmente insopportabile che li spaccherà preferendo poi tornare a piedi nudi, infliggendosi questo dolore quasi per trovare un posto al violento odio di sé provato in quel momento.

Al ritorno da quella giornata, in uno strano italiano, una lingua venuta da altrove e che forse proprio per questo può esprimere la sensazione di non poter essere da nessuna parte, in nessun luogo, Okoko dirà di sentirsi "vomitata dalla terra".

Non poter essere da nessuna parte, in nessun luogo, in nessun corpo, in nessun tempo: né con quella minigonna con cui ostentava sicurezza sotto le mentite spoglie di un 'travestimento' all'occidentale, né in una veste da ragazza 'normale', perché è proprio lì che il senso di inadeguatezza si fa più intollerabile, l'odio di sé si manifesta in maniera devastante e la sensazione di non poter esistere sotto nessuna 'forma', né psichica né culturale, diventa assoluta. Come se non ci fosse un posto per te sulla terra, che ti vomita incessantemente.

E poi qualche giorno dopo, quasi per caso, mentre è seduta sulla sponda del letto a riordinare i vestiti, una quieta attività quotidiana, di quelle abitudini fidate che ti fanno sentire 'a casa', racconterà l'irraccontabile: le violenze subite, lo spaesamento, soprattutto l'impossibilità di credere che davvero la sua famiglia voglia questo da lei. La finestra sul passato come improvvisamente e inopinatamente si è aperta, così si deve richiudere, deve restare sospesa e 
'dislocata' per potere essere mantenuta e non espulsa. Frammento di orrore che contemporaneamente ha fatto irruzione nella mente di chi r-accoglie invece gli affetti devastanti e disorganizzanti che migrano in cerca di un senso.

Un'immagine insostenibile viene letteralmente inoculata, 'messa negli occhi', di un altro affinché il suo apparato psichico possa per primo guardare in quello che tu hai visto, soffrire quello che è rimasto congelato. Forse solo quando si comincia a vedere la propria ferita nello sguardo ferito dell'altro - un altro che si è lasciato ferire nella sua umanità dal disumano - forse solo allora si potrà cominciare a ospitare in sé la propria storia.

Già Tobie Nathan (1990) sottolineava che il migrante soffre soprattutto di una perdita del 'doppio culturale', sorta di 'doppio psichico' che assicura sul fatto che strutturazione interna ed esterna coincidano, ovverosia che la propria strutturazione interna si rispecchi e si reduplichi in quella esterna.

$\mathrm{E}^{\prime}$ solo nelle situazioni migratorie più recenti - che come abbiamo sottolineato comportano uno smarrimento quasi completo delle coordinate autorappresentative originarie - che può emergere quanto questa perdita coinvolga aree psichiche arcaiche, in particolare quelle che strutturano la percezione e la rappresentazione corporea.

Okoko non ha più una terra/corpo in cui dimorare e, come moltissimi migranti adolescenti, cerca di darsi un 'doppio corpo' da utilizzare all'occasione: corpo pubblico da un lato, mimetico e disciplinato, simulacro identificativo declinato come 'corpo seduttivo' all'occidentale, e corpo privato dall'altro, paradossalmente 'inassimilabile' e 'straniero' quanto più ti parla di ciò che più profondamente sei e di cui non puoi appropriarti, corpo parlante una lingua indecifrabile che ignori e che ti ignora. Solo una oscillazione tra questi due corpi potrà consentire quella dislocazione soggettuale necessaria alla mente migrante per proteggersi dal duplice rischio di frantumarsi o di precipitare, poichè, come scrive Thomas Sterns Eliot (1959): "per arrivare dove non sei/ devi fare la strada per la quale non sei".

\section{Sul margine}

Sul margine ci sono lutti da elaborare e scoperte da fare: "Solo ciò che è umano può esserci straniero" scriveva la poetessa polacca Wislawa Szymborska il più autentico riconoscimento di 'umanità' all'altro, e paradossalmente anche a sè stessi, consiste proprio nel potergli riconoscere il diritto di essere straniero, il diritto di resistere e di mantenere un elemento inassimilabile.

Il problema della contemporaneità secondo Marc Augè (2000) sarebbe infatti quello di non riuscire più a costruire I' Altro, a fare esperienza dell'

\footnotetext{
${ }_{6}$ Cf. <https://barbarapanelli.blogspot.com/2016/08/il-salmo-di-wislawa-szymborska.html>.
} 
Altro. Al suo posto paiono invece instaurarsi 'doppi deformati' in cui guardarsi come in perturbanti specchi deformanti. E' quello che accade spesso ai giovani migranti che nel loro slancio mimetico possono talvolta trovarsi ancora più violentemente rigettati, perché cominciano a divenire una involontaria e grottesca caricatura di coloro a cui vogliono a tutti costi 'assimilarsi'. L'inquietante vicinanza può diventare ancora più perturbante dell'inquietante estraneità.

Si può decidere di voltare completamente le spalle all'abisso fingendo che riguardi qualcun altro, o si può essere costretti a fissare lo sguardo sul suo fondo come accade a chi solca il Mediterraneo sui barconi e si perde nel buio liquido della notte in mare aperto, buio che infiltra la mente e la circonda da ogni parte, oppure si può cercare di avanzare sul margine di quell'abisso "cui sempre camminiamo a lato" (Eliot, 1959).

"Non si torna mai da così lontano come da sé stessi" scriveva Tahar Ben Jelloun (1987), e qualche volta non si torna affatto aggiungerei, qualche volta bisogna accontentarsi di sostare sul margine, margine di sé e di un mondo che si possa sentire proprio: quel margine di terraferma da strappare progressivamente al mare con paziente tenacia e dedizione che, nella metafora freudiana dello Zuidersee, rappresenta l'opera della civiltà.

Ma è quel margine, quel piccolo margine, che fa, e farà, tutta la differenza...

\section{Bibliografia}

ABRAHAM, Nicholas; TOROK, Maria. La scorza e il nocciolo. Roma: Borla, 1993. AUGÉ, Marc. Il senso degli altri. Torino: Boringhieri, 2000.

BAUMAN, Zigmut. Modernità liquida. Roma-Bari: Laterza, 2002.

BAUMAN, Zigmut. Vita liquida. Roma-Bari: Laterza, 2006.

DE MICCO, Virginia. Fuori luogo Fuori tempo. L'esperienza dei minori migranti non accompagnati tra sguardo antropologico ed ascolto analitico. Adolescenza e Psicoanalisi, Magi ed. Roma, n. 1, 2019.

DE MICCO, Virginia. Migrare. Sopravvivere al disumano. Rivista italiana di Psicoanalisi, n. 4, 2017.

DE MICCO, Virginia. Le identità nomadi. Identità, migrazioni, fratture narcisistiche. In: COTRUFO, Paolo; POZZI, Rossella (a cura di). Identità e processi di identificazione. Milano: F. Angeli, Milano, 2014a, p. 137-151.

DE MICCO, Virginia. Trapiantare/tramandare. Legami e identificazioni nei transiti migratori. In: De MICCO, Virginia; GRASSI, Ludovica (a cura di). "Soggetti in transito. Etnopsicoanalisi e migrazioni", numero monografico della rivista Interazioni. Clinica e ricerca psicoanalitica su individuo-coppia-famiglia, n. 1 2014b. 
ELIOT, Thomas S. Quattro quartetti. Milano: Garzanti, 1959.

FERENCZI, Sándor. Diario Clinico. Gennaio-Ottobre 1932. Milano: Cortina, 1988.

FREUD, Sigmund. Introduzione al narcisismo. OSF v. 7. Torino: Bollati Boringhieri, 1914.

FREUD, Sigmund. II Perturbante. OSF v. 9. Torino: Bollati Boringhieri, 1919.

FREUD, Sigmund. L'lo e l'Es. OSF, v. 9. Torino: Bollati Boringhieri, 1922.

FRIGESSI CASTELNUOVO, Delia; RISSO, Michele. A mezza parete. Emigrazione Nostalgia Malattia mentale. Torino: Einaudi,1982.

GREEN, André. Il lavoro del negativo. Roma: Borla, 1996.

GUTTON, Philippe. Adolescence et Jihadism. Paris: L'esprit du temps, 2015.

GRINBERG, León; GRINBERG, Rebeca. Psicoanalisi dell'emigrazione e dell'esilio. Milano: Angeli, 1990.

KAES, René. La trasmissione delle alleanze inconsce, organizzatori metapsichici e metasociali. In: AA.VV. Generi e generazioni. Ordine e disordine nelle identificazioni. Milano: Angeli, 2008.

KAES, René. Le alleanze inconsce. Roma: Borla, 2010.

KAES, René. Il Malessere. Roma: Borla, 2013.

JELLOUN, Tahar Ben. Creatura di sabbia. Torino: Einaudi editore, 1987.

NATHAN, Tobie. La follia degli altri. Etnopsichiatria. Firenze: Ponte alle grazie, 1990.

RAISON, Jean Paul. Migrazione. In: Enciclopedia. Torino: Einaudi, 1980.

RISSO, Michele; BÖKER, Wolfgang. Sortilegio e delirio. Psicopatologia delle migrazioni in prospettiva transculturale (a cura di Lanternari, Vittorio; De Micco, Virginia; Cardamone, Giuseppe). Napoli: Liguori, 2000.

ROSOLI, Gianfausto. La condizione sanitaria dell'emigrante. In: Atti del I Convegno internazionale Medicina e Migrazioni. Roma, 9-10 aprile 1988.

RUSSO, Lucio. Destini delle identità. Roma: Borla, 2009.

SAYAD, Abdelmalek. La doppia assenza. Milano: Cortina, 2001.

VAN GENNEP, Arnold. I riti di passaggio. Torino: Bollati Boringhieri, 1909.

WEIL, Simone. La prima radice. Preludio ad una dichiarazione dei doveri verso I'essere umano. Milano, 1990. 